\title{
Isoniazid and Rifampicin Produce Hepatic Fibrosis through an Oxidative Stress-Dependent Mechanism
}

\author{
Ayan Biswas,, ${ }^{1,2}$ Suman Santra,, ${ }^{1,2}$ Debasree Bishnu, ${ }^{1,3}$ Gopal Krishna Dhali, ${ }^{1}$ \\ Abhijit Chowdhury, ${ }^{1,3}$ and Amal Santra ${ }^{1,3}$ \\ ${ }^{1}$ Centre for Liver Research, School of Digestive \& Liver Diseases, Institute of Post Graduate Medical Education \& Research, \\ Kolkata, India \\ ${ }^{2}$ Indiana University School of Medicine, Indianapolis, USA \\ ${ }^{3}$ JCM Centre for Liver Research and Innovations, Kolkata, India
}

Correspondence should be addressed to Amal Santra; asantra2000@yahoo.co.in

Received 30 December 2019; Revised 18 March 2020; Accepted 26 March 2020; Published 23 April 2020

Academic Editor: Dirk Uhlmann

Copyright (C) 2020 Ayan Biswas et al. This is an open access article distributed under the Creative Commons Attribution License, which permits unrestricted use, distribution, and reproduction in any medium, provided the original work is properly cited.

Background \& Aims. Chronic hepatitis (CH) has emerged as a distinct outcome of drug-induced liver injury (DILI). Combination therapy of Isoniazid (INH) and Rifampicin (RMP) which is widely used for prolonged periods can cause acute hepatotoxicity and has been also incriminated in chronic DILI. We sought evidence of the production of hepatic fibrosis on long-term INH-RMP treatment through experiments in BALB/c mice exposed to INH-RMP. Methods. A combined dose of INH (50 mg) and RMP ( $100 \mathrm{mg}$ ) per $\mathrm{kg}$ body weight per day was administered to mice by oral gavage, 6 days a week, for 4 to 24 weeks for the assessment of liver injury, oxidative stress, and development of hepatic fibrosis, including demonstration of changes in key fibrogenesis linked pathways and mediators. Results. Progressive increase in markers of hepatic stellate cell (HSC) activation associated with changes in matrix turnover was observed between 12 and 24 weeks of INH-RMP treatment along with the elevation of liver collagen content and significant periportal fibrosis. These were associated with concurrent apoptosis of the hepatocytes, increase in hepatic cytochrome P450 2E1 (CYP2E1), NADPH oxidase (NOX) activity, and development of hepatic oxidative stress. Conclusions. INH-RMP can activate HSC through generation of NOX-mediated oxidative stress, leading to the development of liver fibrosis.

\section{Introduction}

Conclusive evidence demonstrating a cause-effect relationship between drug hepatotoxicity and development of liver fibrosis is lacking. However, several large and wellcharacterized drug-induced liver injury (DILI) registries, based on prolonged follow-up of well-characterized acute DILI subjects, have shown that chronic hepatitis $(\mathrm{CH})$ can occur as a distinct outcome in DILI [1-5]. Persistent liver function derangements can occur in $5.7 \%$ to $18.9 \%$ of acute DILI subjects $[6,7]$. In addition, histological and clinical features of $\mathrm{CH}$ have been observed in drug hepatotoxicity cohorts and case studies [8-10]. In most of these cases, the frequency of development of chronic DILI increases as the period of observation of the DILI cohort increases. In the absence of a biomarker for precise DILI definition as well as one indication of evolution to chronicity, the entity chronic DILI remains enigmatic despite its significance. In order to bring clarity on the issue, well-designed experimental studies are needed. In this context, looking for evidence of activation of hepatic stellate cells (HSCs) as the key player in $\mathrm{CH}$ and morphological proof for production of liver fibrosis by the drug is important.

Isoniazid (INH) and Rifampicin (RMP) combination therapy is one of the commonest cause to develop acute hepatotoxicity. INH is the primary toxin, and RMP potentiates its toxicity through altered kinetics of metabolites [11, 12]. Recovery from acute hepatitis, clinical or subclinical, generally occurs in clinical settings. Usually, the drugs can be continued thereafter for the originally planned duration of treatment for at least 6 months (often 9-12 months), often in a modified dosage or schedule depending on the presence 
or absence of liver function alterations [11-17]. Overall, INH-RMP combination treatments are associated with overt or indolent and covert hepatocyte functional changes in a significant group of exposed people and hence have the potential to cause activation of HSCs. In view of the prolonged nature of the whole process, this leads to liver fibrosis. Over and above, we have earlier demonstrated, in short term in vivo studies in BALB/c mice, that INH-RMP causes mitochondrial permeability changes and oxidative stress along with hepatocyte apoptosis [18]. Each of these has the potential to activate HSCs.

In the present study, we are seeking experimental evidence for a relationship between prolonged INH-RMP treatment and development of liver fibrosis. We describe here the findings of an in vivo study in BALB/c mice treated with INH-RMP. We wanted to address three pertinent questions in this study: (1) Can INH-RMP cause hepatic fibrosis on long-term exposure? (2) Is there any evidence for HSC activation along with associated alterations in the matrix proteins to substantiate establishment of a profibrogenic milieu on long-term INH-RMP treatment? (3) Does oxidative stress contribute to HSC activation and fibrosis on INH-RMP exposure, with an eye to get mechanistic insights in the process?

\section{Materials and Methods}

2.1. Animals and Their Treatment Schedule. Male mice (BALB/c; 7-8 weeks of age) were purchased from National Center for Laboratory Animal Sciences (NCLAS; Hyderabad, India). Treatment of animals and procedures performed were done in accordance with the guidelines stipulated by the animal ethics committee of the Institute of Post Graduate Medical Education \& Research (I.P.G.M.E.\&R.), Kolkata, India.

Mice $(n=24)$ were treated with a combined dose of INH (50 mg) and RMP (100 mg) per kg body weight per day by gavage, 6 days a week, for 4 to 24 weeks. The dosage regimen was based on our previous report [18]. Control mice received an equal volume of vehicle by gavage in the same schedule of the INH-RMP-treated mice.

2.2. Animal Sacrifice and Sample Collection. During the period of sacrifice, the blood was obtained by cardiac puncture and the serum samples were stored at $-20^{\circ} \mathrm{C}$ for the measurement of alanine aminotransferase (ALT). The liver was removed, rinsed with phosphate-buffered saline (PBS), and divided into four portions: (a) fixed in $10 \%$ buffered formaldehyde (formalin) and embedded in paraffin; (b) homogenized in appropriate buffer(s) and aliquots were frozen at $-70^{\circ} \mathrm{C}$ for biochemical assays; (c) placed in RNA later (from Ambion) solution for RNA expression study; and (d) snap frozen at $-70^{\circ} \mathrm{C}$ for future use.

2.3. Serum Aminotransferases. ALT activity of serum was measured with a commercial kit (DiaSys Diagnostic Systems $\mathrm{GmbH}$, Germany) according to the manufacturer's instruction.
2.4. Hepatic Biochemical Assays. A 10\% liver homogenate was used for the determination of triglyceride (TG) content using a spectrophotometric kit from Sigma Diagnostics (St. Louis, MO, USA), levels of reduced glutathione (GSH), oxidized glutathione (GSSG), thiobarbituric acid reactive substances (TBARs), and protein content [19-22]. Activities of hepatic catalase, glutathione peroxidase (GPx), superoxide dismutase (SOD), cytochrome P450 2E1 (CYP2E1), and NADPH oxidase (NOX) were also determined [23-27]. The collagen content of the liver tissue was measured as described previously [28].

2.5. Histology and TUNEL Assay. Liver tissues embedded in paraffin were cut in sections $(5 \mu \mathrm{m})$ and stained with hematoxylin and eosin $(\mathrm{H} \& \mathrm{E})$ and Sirius red for collagen I detection using standard procedures. Terminal deoxynucleotidyl transferase-mediated deoxyuridine triphosphate nick end labeling (TUNEL) assays were performed using the in situ cell death detection kit (Roche, Germany) according to the manufacturer's instruction. The extent of injury, apoptosis, and fibrosis was evaluated by an investigator, who was blinded to the experimental protocol and graded for steatosis by determining the overall percentage of liver parenchyma containing lipid vacuoles, with $0=$ none, $1=$ mild $(<25 \%)$, $2=$ mild to moderate $(25$ to $<50 \%), 3=$ marked (50 to $<75 \%)$, and $4=$ severe $(>75 \%)$ [29]. Inflammation was graded by the presence or absence of inflammatory cells, with 0 =absent, $1=$ minimal or focal occasional single clusters of inflammatory cells present in a few microscopic fields, $2=$ mild inflammation, $3=$ moderate inflammation, and $4=$ marked inflammation [30]. The pattern of fibrosis was graded with $0=$ none, $1=$ portal fibrosis, $2=$ periportal fibrosis or rare septa, $3=$ septal fibrosis and architectural distortion but not true cirrhosis, and $4=$ cirrhosis, widespread fibrosis, and hepatocyte nodule formation [31].

2.6. Immunostaining. Immunohistochemistry of $\alpha$-smooth muscle actin ( $\alpha$-SMA) was performed from the paraffinembedded sections of the liver. Briefly, deparaffinized liver sections were washed in deionized water for 1 minute and in PBS for 5 minutes, followed by permeabilization in $0.1 \mathrm{M}$ citrate buffer and then blocked using PBS with 3\% bovine serum albumin (BSA). The liver section was then incubated with Cy3 conjugate $\alpha$-SMA antibody (C6198; Sigma) at $4^{\circ} \mathrm{C}$ overnight. After washing, the nuclei were stained with Hoechst (Sigma; 33270) for 5 minutes, washed with PBS, and mounted using Prolong Gold Antifade reagent (Invitrogen; P 36934). Slides were examined by confocal microscopy (Leica, TCS SPE; Germany).

2.7. RNA Isolation and Real-Time Quantitative PCR. Total RNA from liver tissue was obtained using the TRIzol Reagent (Invitrogen, Carlsbad, CA). A High-Capacity cDNA Reverse Transcription kit (Applied Biosystems) was used to generate cDNA from extracted RNA. Primers used for quantitative real-time polymerase chain reaction (qRT-PCR) are shown in Table 1. qRT-PCR was carried out on cDNA using StepOnePlus thermocycler (ABI), primer sets, and SYBR ${ }^{\circledR}$ green PCR master mix (Applied Biosystems) according to the 
TABLE 1: PCR primers.

\begin{tabular}{|c|c|c|}
\hline $\begin{array}{l}\text { Primer } \\
\text { NOX1 }\end{array}$ & Forward & $\begin{array}{c}\text { Primer sequence } \\
5^{\prime} \text {-CTGACAAGTACTATTACACGAGA-3 }\end{array}$ \\
\hline NOX1 & Reverse & $5^{\prime}$-CATATATGCCACCAGATTAGGGA-3' \\
\hline NOX2 & Forward & 5' -CTTTCTCAGGGGTTCCAGTG-3' \\
\hline NOX2 & Reverse & 5'-TCTTCCAAACTCTCCGCAGT-3' \\
\hline NOX4 & Forward & $5^{\prime}$-TGAGGAGTCACTGAACTA-3' \\
\hline NOX4 & Reverse & $5^{\prime}$-TGACTGAGGTACAGCTGGA-3' \\
\hline $\mathrm{p} 22^{\text {phox }}$ & Forward & $5^{\prime}$-TGGACGTTTCACACAGTGGT-3' \\
\hline $\mathrm{p} 22^{\text {phox }}$ & Reverse & $5^{\prime}$-TAGGCTCAATGGGAGTCCAC-3' \\
\hline $\mathrm{p} 47^{\text {phox }}$ & Forward & $5^{\prime}$-CACCGAGATCTACGAGTTCCA-3' \\
\hline $\mathrm{p} 47^{\text {phox }}$ & Reverse & $5^{\prime}$-TGTCAAGGGGCTCCAGATAG-3' \\
\hline p67 $7^{\text {phox }}$ & Forward & $5^{\prime}$-GCCACAGTCATGTTCAATGG-3' \\
\hline p67 $7^{\text {phox }}$ & Reverse & $5^{\prime}$-ACAAAAGCCTTCGGGAAAAT-3' \\
\hline PDGF-R $\beta$ & Forward & $5^{\prime}$-AGCACCTACCTGCCTCTGAA-3' \\
\hline PDGF-R $\beta$ & Reverse & $5^{\prime}$-GCACGGCAGTGTAGAGAACA-3' \\
\hline$\alpha$-SMA & Forward & $5^{\prime}$-ACTACTGCCGAGCGTGAGAT-3' \\
\hline$\alpha$-SMA & Reverse & $5^{\prime}$-AAGGTAGACAGCGAAGCCAA-3' \\
\hline TGF- $\beta$ & Forward & $5^{\prime}$-GTCAGACATTCGGGAAGCAG-3' \\
\hline TGF- $\beta$ & Reverse & $5^{\prime}$-GCGTATCAGTGGGGGTCA-3' \\
\hline COL1A1 & Forward & $5^{\prime}$-GAAACCCGAGGTATGCTTGA-3' \\
\hline COL1A1 & Reverse & $5^{\prime}$-GACCAGGAGGACCAGGAAGT-3' \\
\hline TIMP-1 & Forward & $5^{\prime}$-ATCAATGCCTGCAGCTTCTT-3' \\
\hline TIMP-1 & Reverse & 5'-ATCTCCAAGTGCACAAGCCT-3' \\
\hline MMP2 & Forward & $5^{\prime}$-GAGAAGGATGGCAAGTATGGC-3' \\
\hline MMP2 & Reverse & 5'-GCGGTCTCGGGACAGAATCC-3' \\
\hline MMP9 & Forward & $5^{\prime}$-GAGTGGACGCGACCGTAGTTGG-3' \\
\hline MMP9 & Reverse & $5^{\prime}$-GTACATGAGCGCTTCCGGCACGC-3' \\
\hline$\beta$-Actin & Forward & $5^{\prime}$-TGGAATCCTGTGGCATCCATGAAAC-3' \\
\hline$\beta$-Actin & Reverse & $5^{\prime}$-TAAAACGCAGCTCAGTAACAGTCCG-3' \\
\hline
\end{tabular}

NOX = NADPH oxidase; PDGF-R $\beta=$ platelet-derived growth factor receptor $\beta ; \alpha$-SMA $=\alpha$-smooth muscle actin; TGF- $\beta=$ transforming growth factor $\beta$; COL1A1 = collagen $1 \mathrm{~A} 1$; TIMP = tissue inhibitor of matrix metalloproteinase; $\mathrm{MMP}=$ matrix metalloproteinase.

manufacturer's instructions. Data were normalized to the expression of $\beta$-actin, a housekeeping gene.

2.8. Caspase Activity. The activity of caspase 3 was determined in liver homogenates by measuring proteolytic cleavage of the specific fluorogenic substrate DEVD-AFC (AspGlu-Val-Asp) (AFC: 7-amino-4-trifluoromethyl coumarin, respectively; BioVision). The results are expressed as the percentage of control.

2.9. Cytokine Quantification. Hepatic transforming growth factor $\beta$-1 (TGF- $\beta 1$ ) levels were evaluated by enzymelinked immunosorbent assay (ELISA) using quantikine kits of R\&D system.
2.10. Western Blotting. For western blotting, tissue lysate was prepared using RIPA buffer (Cell signaling Technology, USA) with protease inhibitors cocktail (Roche Diagnostics, Mannheim, Germany). The lysates were centrifuged at $14,000 \times \mathrm{g}$ for $20 \mathrm{~min}$ at $4^{\circ} \mathrm{C}$. The protein content of the supernatant was determined with the Bradford protein assay (Sigma, USA). Forty micrograms protein was resolved on sodium dodecyl sulfate-polyacrylamide gel electrophoresis (SDS-PAGE) after denaturing in sample buffer and transferred onto polyvinylidene fluoride membranes (PVDF; Thermo Fisher Scientific, USA). After blocking with 5\% BSA, the blots were probed with the following antibodies: mouse monoclonal $\alpha$-SMA (1:300; Santa Cruz Biotechnology), mouse monoclonal beta actin $(1: 1000$; Santa Cruz 
TABLE 2: INH and RMP cotreatment and hepatic oxidative stress.

\begin{tabular}{|c|c|c|c|c|c|c|}
\hline \multirow{2}{*}{ Parameters } & \multicolumn{6}{|c|}{ Groups } \\
\hline & Cont $_{4}$ & $\mathrm{INH} \mathrm{RMP}_{4}$ & Cont $_{12}$ & $\mathrm{INH}_{-} \mathrm{RMP}_{12}$ & Cont $_{24}$ & ${\mathrm{INH}-\mathrm{RMP}_{24}}$ \\
\hline \multicolumn{7}{|l|}{ Cytosolic fraction $(n=8)$} \\
\hline SOD (Sp activity/min/mg protein) & $125.83 \pm 5.48$ & $142.18 \pm 23.13^{*}$ & $123.46 \pm 7.14$ & $72.87 \pm 17.02^{*, \#}$ & $121.04 \pm 11.61$ & $55.06 \pm 10.74^{*, \sharp}$ \\
\hline GPx (Sp activity/min/mg protein) & $6.44 \pm 0.66$ & $7.32 \pm 0.34^{*}$ & $6.24 \pm 0.59$ & $4.61 \pm 0.34^{*, \#}$ & $6.86 \pm 0.62$ & $2.56 \pm 0.22^{*, \ddagger}$ \\
\hline Catalase (Sp activity/min/mg protein) & $5.93 \pm 0.54$ & $6.51 \pm 0.31^{*}$ & $6.06 \pm 0.39$ & $4.65 \pm 0.38^{*, \#}$ & $6.41 \pm 0.66$ & $2.67 \pm 0.36^{*, \ddagger}$ \\
\hline
\end{tabular}

Mice were cotreated with $50 \mathrm{mg}$ INH and $100 \mathrm{mg} \mathrm{RMP} / \mathrm{kg}$ body weight by oral gavage for 6 days a week for 4 , 12 , and 24 weeks, respectively, and were subsequently sacrificed. The results are expressed as the mean $\pm \mathrm{SD}$ of 8 mice in each group. ${ }^{*} p<0.05$ vs. respective control; ${ }^{*} p<0.05$ vs. INH-RMP

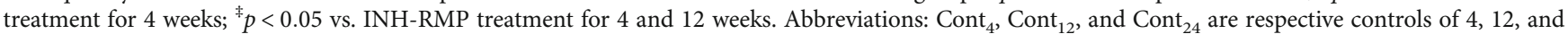
24 weeks while INH-RMP ${ }_{4}$ INH-RMP 12 , and INH-RMP 24 are INH-RMP-treated animals for 4, 12, and 24 weeks, respectively.

Biotechnology), mouse monoclonal collagen $1 \mathrm{~A} 1 \quad(1: 300$; Santa Cruz Biotechnology), mouse monoclonal anti Bax (1:300; Santa Cruz Biotechnology), mouse monoclonal Bcl2 (1:300; Santa Cruz Biotechnology), and mouse monoclonal cytochrome c (1:300; Santa Cruz Biotechnology). The immune complexes were visualized using the enhanced chemiluminescence (ECL) method.

2.11. Statistical Analysis. Results are expressed as the mean \pm SD. Student's $t$-test was used to evaluate statistical differences between groups, and the Mann-Whitney test was used for the comparison of histological findings. A $p$ value less than 0.05 was considered significant.

\section{Results}

3.1. Oxidative Stress and Liver Injury. Liver injury due to prolonged INH and RMP cotreatment in mice was assessed by measuring serum ALT level as well as histological evaluation of liver specimens. We observed a trend towards increase in serum ALT level at 4, 12, and 24 weeks of INH and RMP cotreatment $(52.80 \pm 3.91 \mathrm{IU} / \mathrm{L}$ at 4 weeks of treatment compared to $26.40 \pm 5.41 \mathrm{IU} / \mathrm{L}$ in control mice, $p<0.01 ; 63.75$ $\pm 4.45 \mathrm{IU} / \mathrm{L}$ at 12 weeks of treatment vs. $26.60 \pm 2.26 \mathrm{IU} / \mathrm{L}$ in control mice, $p<0.001$; and $55.50 \pm 5.50 \mathrm{IU} / \mathrm{L}$ at 24 weeks of INH and RMP cotreatment against control $24.66 \pm 1.52$ IU/L, $p<0.001)$. We therefore assessed activities of hepatic antioxidant enzymes like SOD and other GSH-related enzymes in mice cotreated with INH and RMP. Following 4 weeks of INH-RMP treatment, activities of GPx and catalase were significantly increased (Table 2). From 12 weeks and onwards of cotreatment of INH and RMP to mice, the activities of hepatic SOD, GPx, and catalase were significantly decreased (Table 2), indicating persistent oxidative stress in the liver.

\subsection{Prolonged INH-RMP Treatment Causes Hepatic Fibrosis.} Histological changes in the liver due to prolonged INH-RMP treatment included fat infiltration, necrosis, inflammation, and, most importantly, hepatic fibrosis. Steatosis was pronounced all through the 24 weeks of exposure (Figures 1(a) and $1(\mathrm{~b}))$.

Liver TG levels increased also on INH-RMP treatment (Figure 1(c)) in a temporal sequence that paralleled hepatic steatosis. Minimal inflammation and absence of fibrosis were observed at 4 weeks, whereas mild to moderate inflammation and mild portal fibrosis in the liver were evident at 12 weeks (Figures 1(d) and 1(e)). There was a progressive increase in the inflammatory cell infiltration, and the extent of periportal fibrosis was observed in the liver of mice treated with INHRMP at 24 weeks (Figures 1(d) and 1(e)).

INH-RMP treatment induced collagen 1A1 (COL1A1) mRNA (Figure 2(a)) expressions at 12 weeks, which showed further increase at 24 weeks (Figure 2(a)). Changes in hepatic hydroxyproline content, an amino acid specially contained in collagen, paralleled the induction of COL1A1 mRNA expressions, at different time periods of INH and RMP cotreatment (Figure 2(b)).

3.3. INH-RMP Treatment Is Associated with Stellate Cell Activation and Matrix Remodeling. We examined the number of activated HSCs by immunohistochemistry of $\alpha$-SMA using a confocal microscope at different time periods of INH-RMP treatment to seek evidence for activation of HSCs (Figure 2(c)).

The number of activated HSCs progressively increased over time and showed a relationship with the duration of INH-RMP treatment in mice (Figure 2(d)). In addition, we observed an increase in $\alpha$-SMA mRNA expression in the liver tissue, beginning at 12 weeks of INH-RMP treatment (Figure 2(e)).

Proliferation and activation status of HSCs were assessed through expression of candidate molecule platelet-derived growth factor receptor $\beta$ (PDGF-R $\beta)$. As shown in Figure 3(a), PDGF-R $\beta$ mRNA expression showed incremental increase after 12 and 24 weeks, consistent with the activation of HSCs.

Next, we assessed the tissue inhibitor of matrix metalloproteinase-1 (TIMP-1) expression in the liver tissues of mice and observed the induction of TIMP-1 mRNA after 12 and 24 weeks of INH-RMP treatment (Figure 3(b)). TIMP-1 is synthesized and secreted by activated HSCs in response to fibrogenic cytokines, in particular to transforming growth factor $\beta 1$ (TGF- $\beta 1$ ) [32]. There was a significant increase in hepatic TGF- $\beta$ protein levels after 24 weeks of INH-RMP treatment compared to control mice (Figure 3(d)), a finding that was also confirmed by mRNA expression for TGF- $\beta 1$ (Figure 3(c)).

Finally, we also assessed mRNA expression of matrix metalloproteinases 2 and 9 (MMP2 and MMP9), matrix 


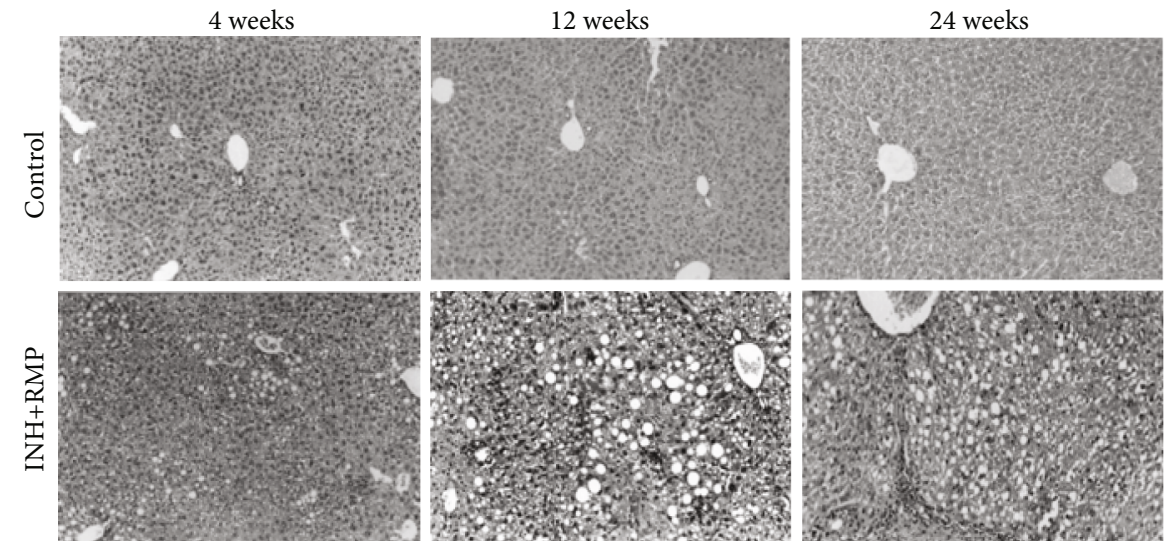

(a)

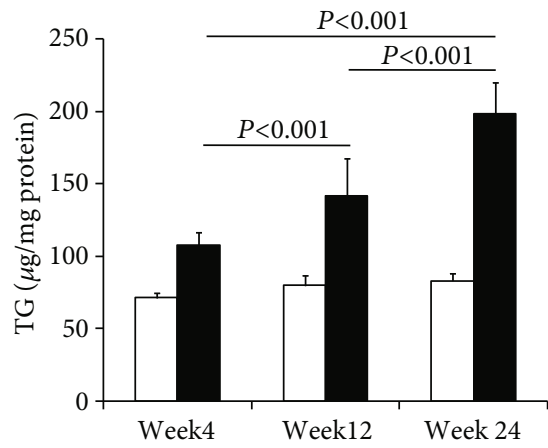

(c)

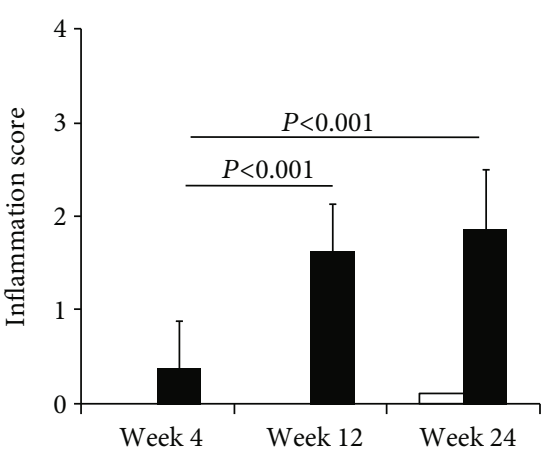

(d)

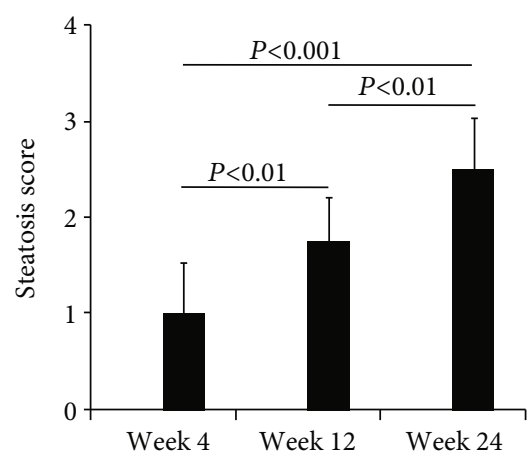

(b)

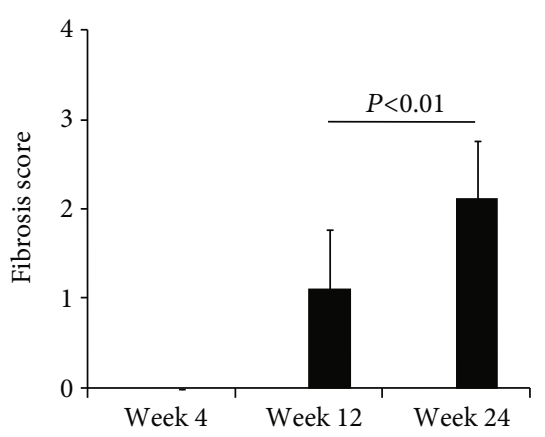

(e)

FIgURE 1: INH and RMP induced chronic liver injury in mice. Paraffin-embedded liver sections were stained with H\&E, examined blindly, and graded for steatosis, inflammation, and fibrosis as described in Materials and Method. (a) H\&E staining of control and INH and RMP treatment mice for 4, 12, and 24 weeks, respectively. Magnification: 10x. (b) Hepatic steatosis score, (c) liver triglyceride content of the control and experimental groups, (d) inflammation score in the liver in different weeks after INH-RMP treatment, and (e) fibrosis score are depicted as the mean \pm SD of eight mice per group. White bars are for control while black bars are for INH+RMP-treated mice.

remodeling-associated molecules [33] which showed marked upregulation between 12 and 24 weeks of INH-RMP treatment (Figures 3(e) and 3(f)).

\subsection{Increased Oxidative Stress Is Related with Hepatic} Fibrosis in Long-Term INH-RMP Treatment. During metabolism of INH-RMP, significant stress is being generated within the hepatocytes by the formation of reactive oxygen species (ROS) which is a potential mediator of HSC activation.

We evaluated the oxidative stress markers that revealed a significant decrease in hepatic GSH level (Figure 4(a)) and an increase of lipid peroxidation as evident by thiobarbituric acid reactive substance (TBAR) level (Figure 4(c)). All these data suggested the development of oxidative stress during INH-RMP treatment.

In view of the critical role of CYP2E1 in oxidative stress in INH-mediated hepatotoxicity, we measured hepatic CYP2E1 activity. This showed progressive increase from 4 to 24 weeks of INH-RMP treatment (Figure 4(b)).

Parallel to CYP2E1 activity, NOX activity increased progressively with the duration of INH-RMP treatment (Figure 4(d)). To confirm further that INH-RMP activates
NOX in a murine liver, a real-time mRNA expression study of the NOX subunits was performed which revealed a significant increase in the expression of different subunits (Figure 4(e)).

\subsection{INH-RMP Treatment Increases Apoptosis of Hepatocytes} in a Manner Chronologically Relevant to Hepatic Fibrosis. In the current context of long-term exposure to INH-RMP, we studied the incidence of apoptosis in the liver tissue. The number of apoptotic cells showed a gradual increase with INH-RMP treatment from 12 to 24 weeks, expressed as an increase in the percentage of TUNEL positive nuclei (Figure 5(a)). Next, we observed a time-dependent decrease in the expression of the specific antiapoptotic protein $\mathrm{Bcl}-2$ (Figure 5(b)), which has been shown to act on the mitochondria and prevent the release of cytochrome $\mathrm{c}$ and subsequent caspase activation [34].

As illustrated in Figure 5(b), a progressive increase in translocation of cytochrome $\mathrm{c}$ in the cytosol and increased proapoptotic Bax expression at different time points of INH-RMP treatment by western blot were consistent with the findings from the TUNEL assay. To confirm these findings, caspase 3 activity was estimated in the cytosolic fraction 


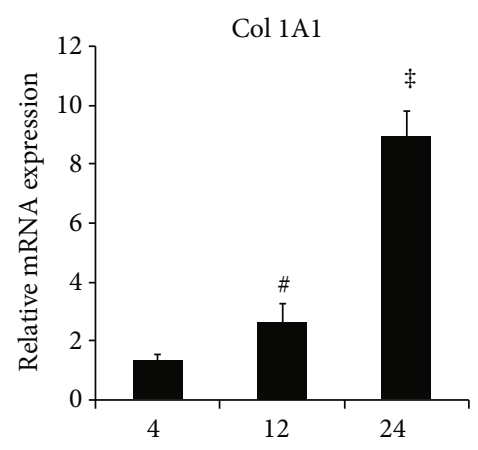

Weeks of INH+RMP treatment

(a)
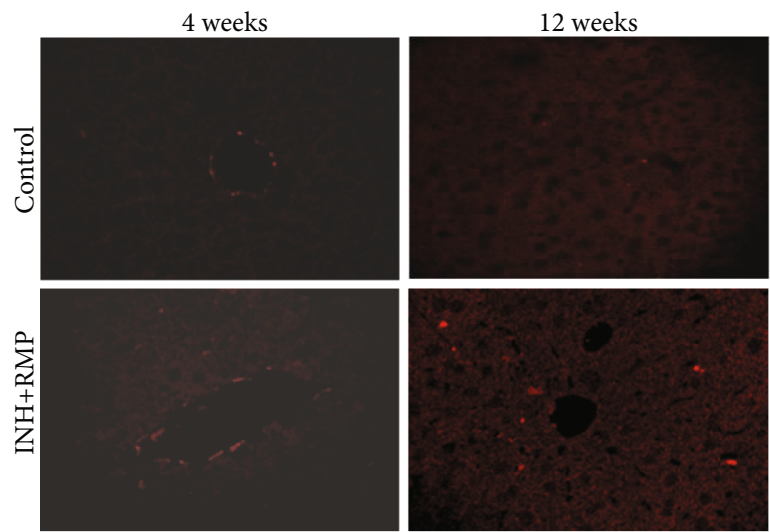

(c)

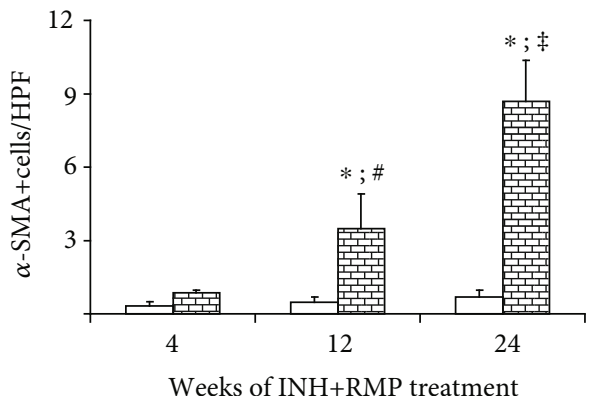

(d)

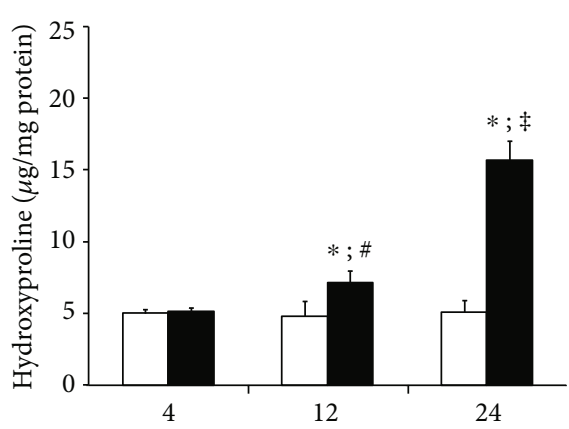

Weeks of INH+RMP treatment

(b)
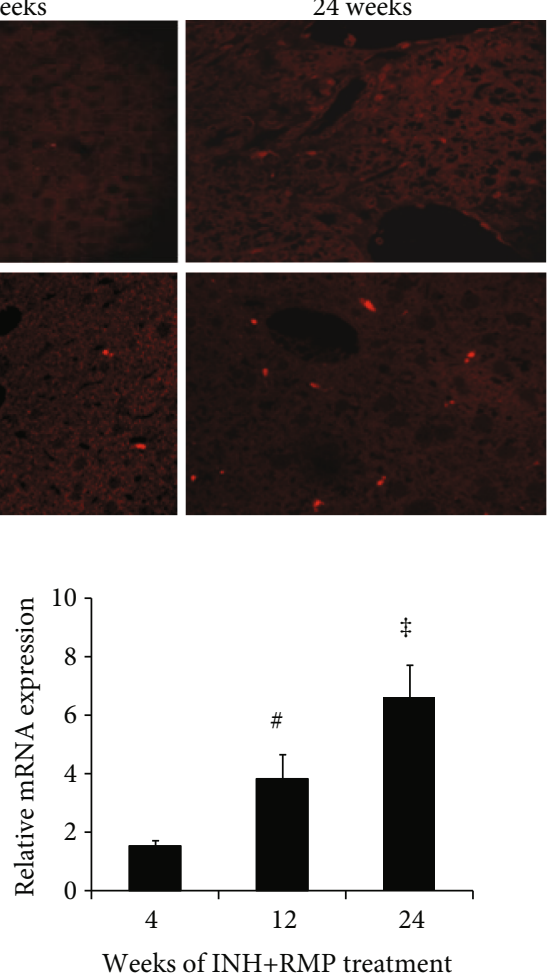

(e)

FIGURE 2: INH and RMP induced HSC and collagenesis. Activation of stellate cells and increased hepatic collagenesis at different time periods after INH and RMP treatment. (a) mRNA expression of CollA1 in the liver at different time points (4-24 weeks) of INH and RMP treatment. (b) Hepatic hydroxyproline content of INH and RMP-treated mice at different time periods. White bars are for control while black bars are for INH+RMP-treated mice. (c) Confocal laser scanning fluorescence microscopy of $\alpha$-SMA demonstrates the localization of activated HSCs in a mouse liver. Magnification: 40x. (d) The numbers of activated HSCs as identified by the immunohistochemistry of $\alpha$-SMA from the paraffin sections of the liver tissues are shown graphically. $\square$ is the control, and 国 is the INH+RMP-treated mouse. (e) mRNA expression of $\alpha$-SMA in the liver at 4,12, and 24 weeks of INH and RMP treatment. The results are expressed as the mean \pm SD of 8 mice per group. $\left({ }^{*} p<0.05\right.$ versus the vehicle-treated control group; ${ }^{*} p<0.05$ versus INH and RMP-treated mice for 4 weeks; ${ }^{*} p<0.05$ versus INH and RMP-treated mice for 4 and 12 weeks in (a), (b), (d), and (e)).

of the mouse liver and a progressive increase was revealed in INH-RMP-treated mice from 12 to 24 weeks (Figure 5(c)) compared to control mice.

The increased expression of proapoptotic Bax molecules strongly suggests that INH-RMP treatment causes cell death mediated by the mitochondrial apoptotic pathways.

\section{Discussion}

We show here that INH-RMP treatment in long term in a mouse model can lead to HSC activation and liver fibrosis, acting through liver cell injury mechanisms that involve NOX-dependent oxidative stress and apoptosis of hepatocytes. 


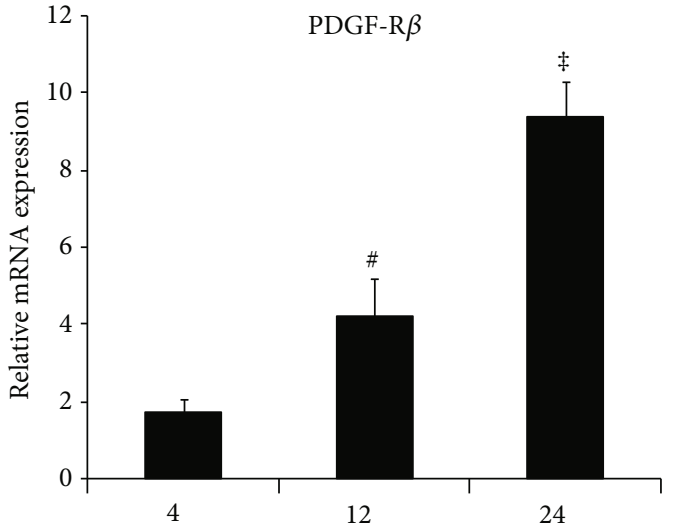

(a)

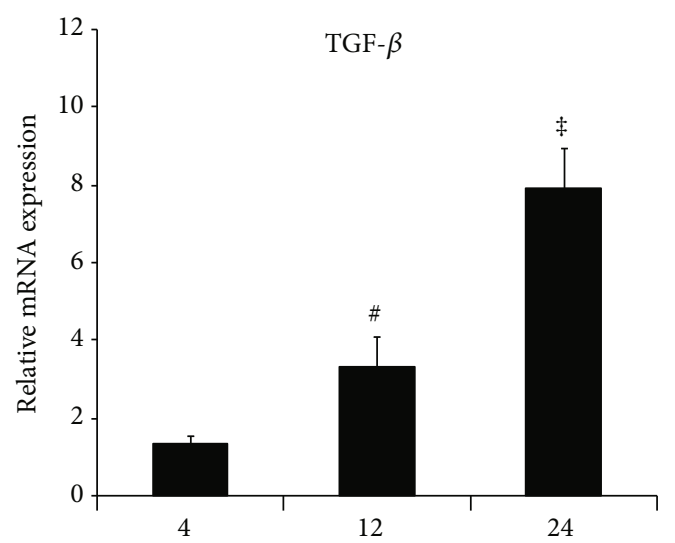

(c)

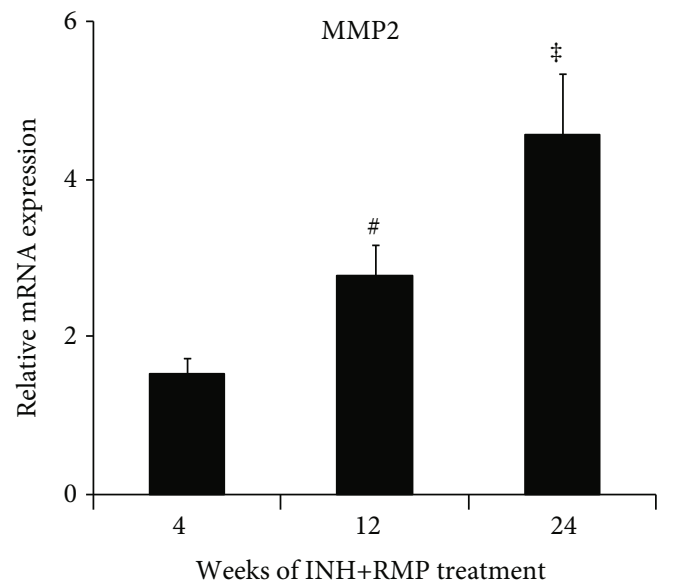

(e)

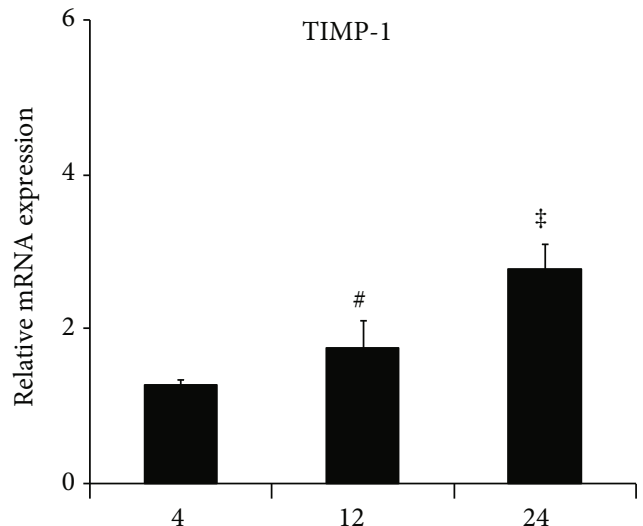

(b)

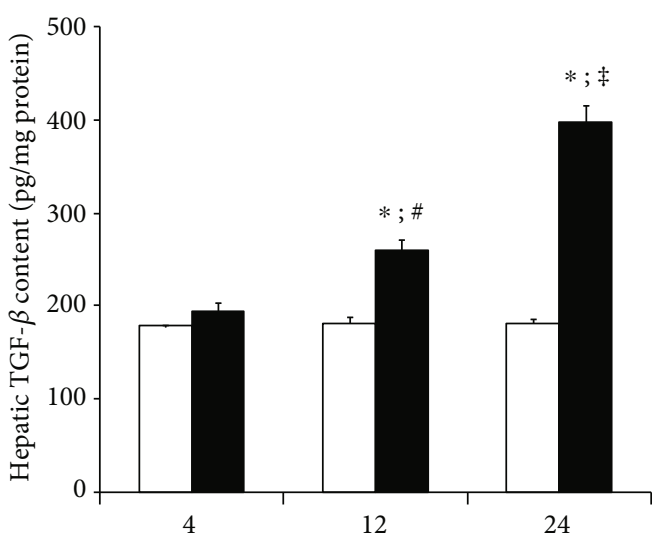

(d)

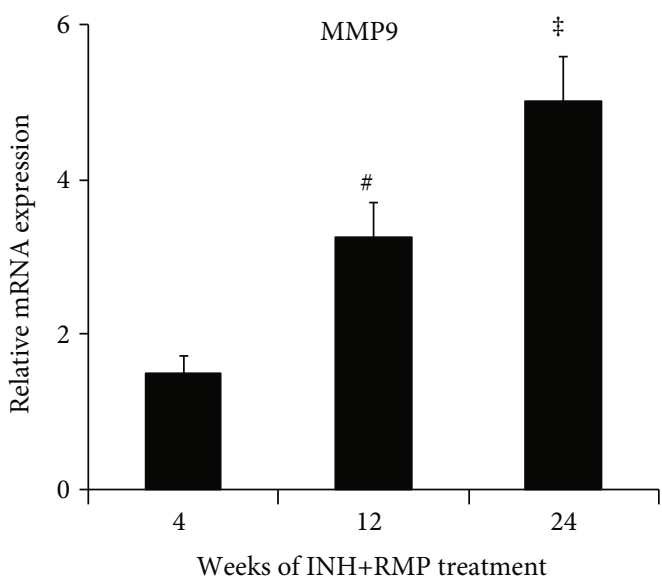

(f)

FIGURE 3: INH-RMP treatment and liver fibrosis in mice. Quantitative RT-PCR demonstrates enhanced expression of (a) PDGF-R $\beta$, (b) TIMP-1, (c) TGF- $\beta$, (e) MMP2, and (f) MMP9 in liver tissues during long-term INH-RMP treatment. ${ }^{*} p<0.05$ versus INH and RMPtreated mice for 4 weeks; ${ }^{*} p<0.05$ versus INH and RMP-treated mice for 4 and 12 weeks. (d) Hepatic TGF- $\beta$ protein level of mice treated with INH-RMP for different time periods. Hepatic TGF- $\beta$ content was quantified by ELISA. The results were expressed as the mean \pm SD of 8 mice per group. ${ }^{*} p<0.05$ versus the vehicle-treated control group; ${ }^{\#} p<0.05$ versus INH and RMP-treated mice for 4 weeks; ${ }^{*} p<0.05$ versus INH and RMP-treated mice for 4 and 12 weeks. White bar indicates only vehicle-treated control mice, while black bar indicates INH-RMP-treated mice.

Our experiments provide evidence in support of the emerging clinical data for chronic DILI [1-7]. INH caused $2.7 \%$ of the chronic DILI in the DILIN data base and is an important component of $\mathrm{CH}$-producing agents in DILI [6,
35]. In this study, we raised two research questions: (a) can INH-RMP produce hepatic fibrosis on long-term exposure, as is commonly used in clinical practice? (b) How can INH-RMP connect with the liver cell injury-repair 


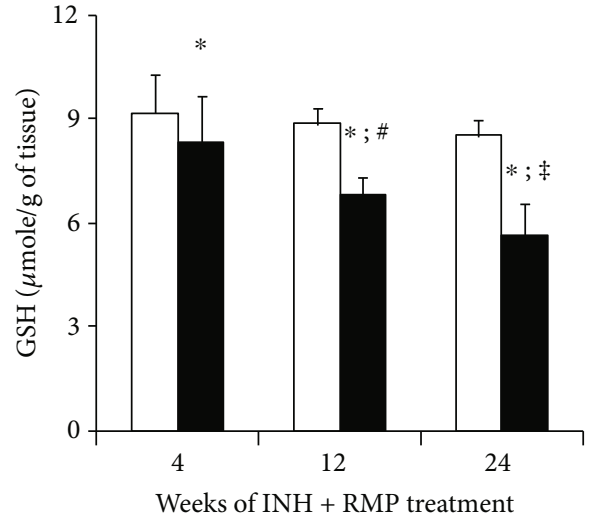

(a)

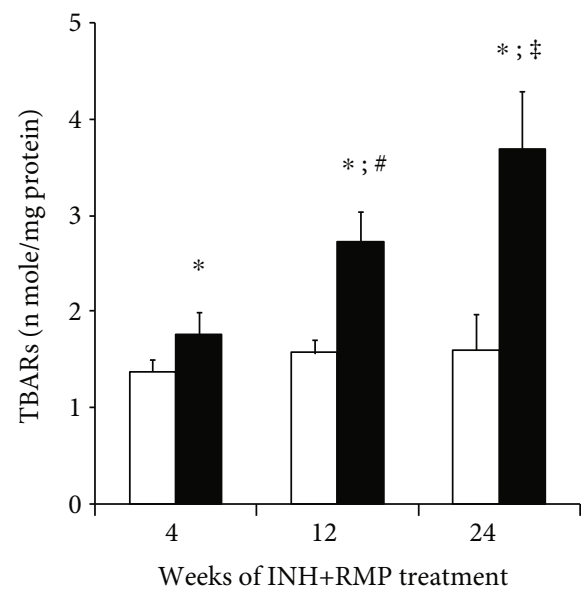

(c)

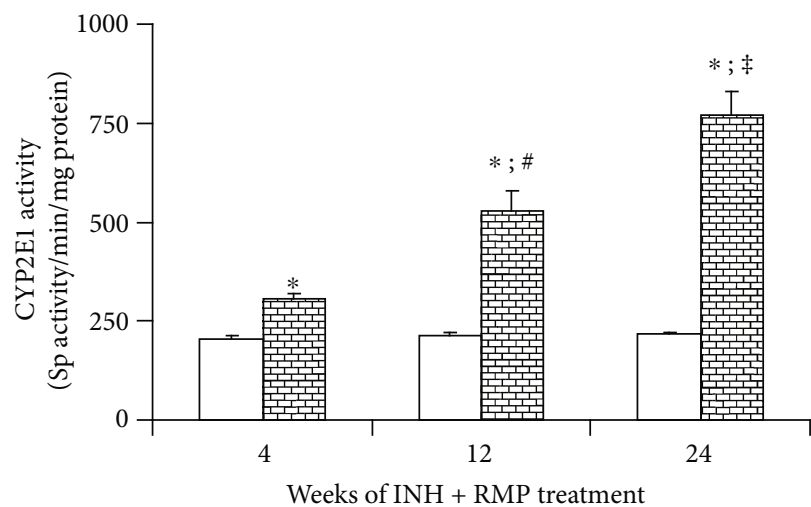

(b)

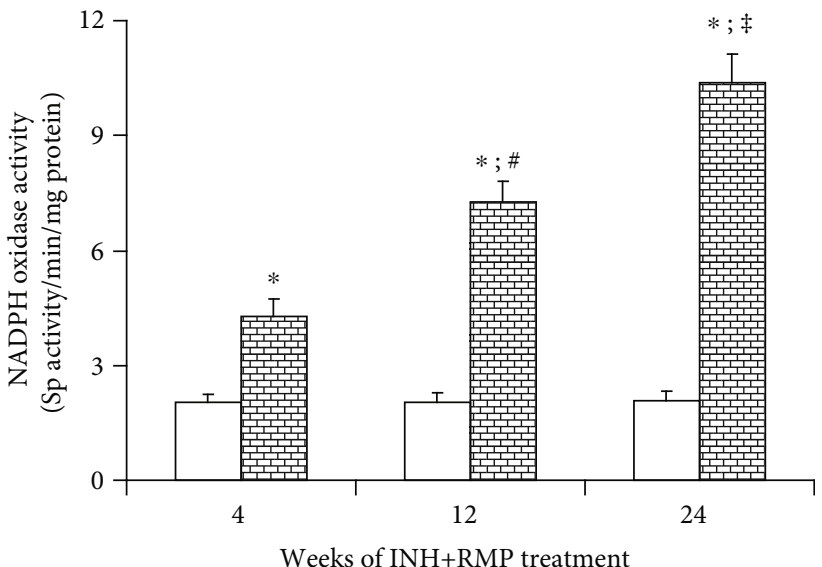

(d)

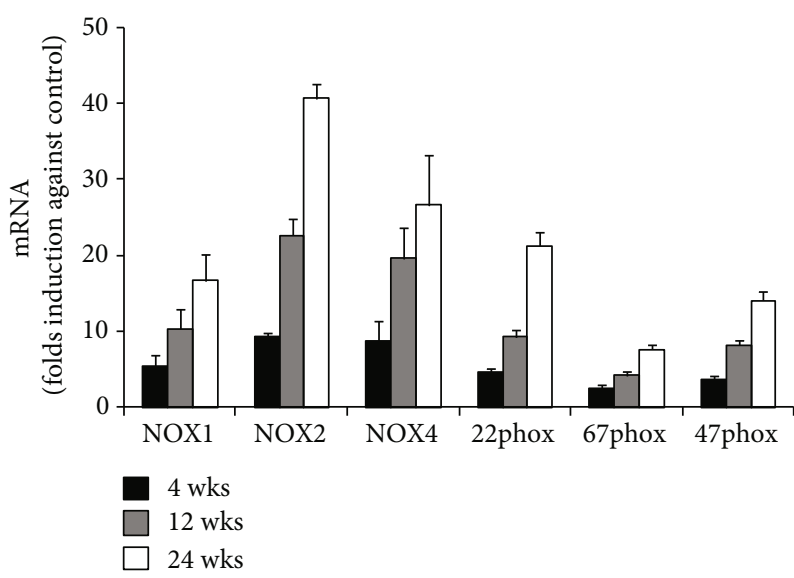

(e)

FIgURE 4: INH and RMP treatment and hepatic oxidative stress. (a) Hepatic GSH and (c) TBAR content in control (white bar) and INH+RMP-treated (black bar) mouse at different weeks. Loss of hepatic GSH content (a) and increased TBAR content (c) in the liver was used as a marker of hepatic oxidative stress. (b) Hepatic CYP2E1 activity in mice. (d) Hepatic NOX activity in mice, for (b) and (d). $\square$ is the control, and 圄 is the INH+RMP-treated mouse; (e) mRNA expression of different isoforms of NOX. Data (a-e) are the means \pm SD of 8 mice per group $\left({ }^{*} p<0.05\right.$ versus vehicle-treated control group; ${ }^{*} p<0.05$ versus INH and RMP-treated mice for 4 weeks; ${ }^{*} p<0.05$ versus INH and RMP-treated mice for 4 and 12 weeks in (a)-(d)).

mechanisms, including oxidative stress, apoptosis of hepatocytes, and HSC activation pathways in producing liver fibrosis?
Given the exploratory nature of the primary research question, we designed in vivo mouse experiments of longterm INH-RMP exposure to seek relevant morphological 


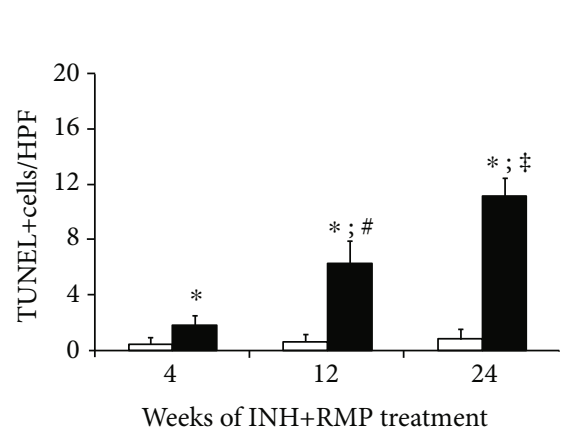

(a)

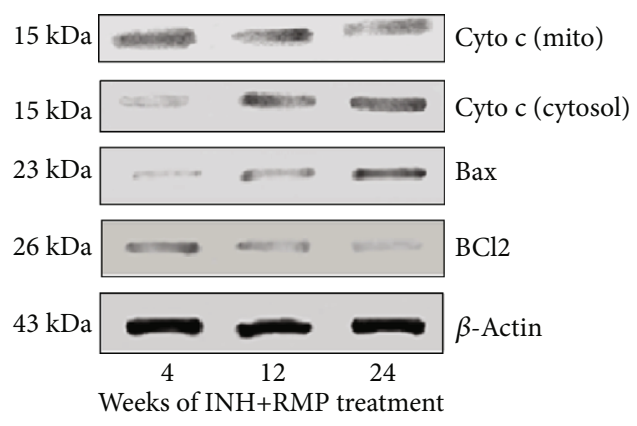

(b)

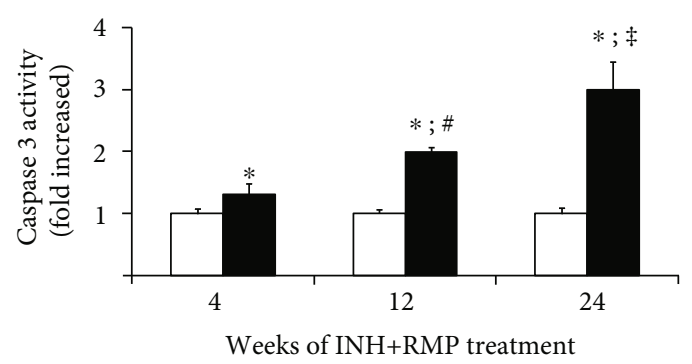

(c)

FIGURE 5: Long-term INH-RMP treatment increases apoptosis of the hepatocytes in mice. (a) Apoptosis in the liver sections was assessed by TUNEL assay. Eight different fields from each liver section were observed, and the mean value was plotted graphically. (b) Western blot analysis for cytochrome c, Bax, and Bcl2 from liver extracts from INH-RMP-treated mice at the end of 4, 12, and 24 weeks. (c) Caspase 3 activity of liver extract was determined using a fluorometric assay with Ac-DEVD AFC, in control and INH and RMP-treated mice after 4,12 , and 24 weeks. Results are presented as the fold increase from control values. The results of (a) and (c) were expressed as the mean \pm SD of 8 mice per group $\left({ }^{*} p<0.05\right.$ versus the vehicle-treated control group; ${ }^{*} p<0.05$ versus INH and RMP-treated mice for 4 weeks; ${ }^{\ddagger} p<0.05$ versus INH and RMP-treated for 4 and 12 weeks). White bar indicates only vehicle-treated control mice, while black bar indicates INH-RMP-treated mice.

and functional evidence in this respect. This approach provides robust data to suggest existence of a profibrogenic state in the liver on long-term exposure to INH-RMP.

In the in vivo study, we used INH-RMP combinations in order to capture the real life scenario in antitubercular therapy where they are used together for at least 6 months. INH-RMP combination therapy is the most common cause of acute DILI and drug-induced acute liver failure in India $[36,37]$. Of the two, INH is the primary hepatotoxic drug in such combinations and RMP modifies the kinetics of toxic metabolite generation through its ability to induce microsomal enzymes. RMP, therefore, primarily plays a role in potentiating INH hepatotoxicity $[12,13,18]$. The doses of INH and RMP used in the present study are about 10 times the human doses on a milligram per kilogram basis; however, they may be equivalent to the human dose but on a body surface area basis [18].

An intriguing aspect of the histology was macrovesicular steatosis along with inflammatory cell infiltration in the early stages of INH-RMP exposure even when fibrosis has already appeared. We have also observed pericellular fibrosis on prolonged therapy - the fibrosis pattern that correlates with steatohepatitis. Histology in INH-RMP hepatotoxicity has been assessed mostly in the setting of acute liver failure and is characterized by varying amounts of necrosis and inflammation. Prominent steatosis has been observed in some human studies of nonliver failure hepatotoxicity $[38,39]$. Previously, we have demonstrated that INH-RMP combination causes acute hepatotoxicity through mitochondrial dysfunction, steatosis, and hepatocyte apoptosis [18]. Progressive increase of cell death and inflammation in the liver as observed in the present study are the characteristic features that are associated with chronic liver injury leading to the progression of the development of fibrosis. Cell death is the primary precipitating event that triggers activation of inflammatory and fibrogenic signals. In the current experiments, we observed CYP2E1-dependent and NOX-mediated oxidative stress along with apoptosis increasing linearly over a period of prolonged exposure. Oxidant stress stimulates apoptosis, and we could document an increase in caspase 3 , cytoplasmic translocation of cytochrome $c$, and reciprocal expressions of the proapoptotic Bax and the antiapoptotic $\mathrm{BCl} 2$ proteins in the current study, indicating the previously described mitochondrial pathways of apoptosis to be active even during the prolonged therapy periods. Further, we found increased expression of NOX that produces ROS and stimulates HSCs, over the entire duration of experiments, suggesting a nonmitochondrial pathway of oxidative stress generation also to be active. Apart from the conventional phagocytic NOX2, the nonphagocytic NOX4 was progressively expressed in the liver due to INH-RMP treatment.

In the context of HSC activation, it is important that both oxidative stress and hepatocyte apoptosis are potent mitogens for HSCs [40-42]. We, interestingly, found a steady 
time sequence relationship of the events that led to liver fibrosis. In the present study, we have seen the activation of the HSCs that depend largely on oxidative stress. Liver fibrosis results from deposition of type I collagen with simultaneous inhibition of its degradation. In the present study, we observed increased TIMP1 mRNA expression in the liver due to prolonged exposure of INH-RMP treatment in mice. TIMP1 is synthesized and secreted by activated HSCs under the influence of TGF- $\beta 1$, which is also increased in the liver of mice due to INH-RMP treatment in the present study. Thus, progressive building up of oxidative stress over time was correlated with expression of HSC activation and proliferation markers starting 12 weeks of exposure of INH-RMP. This, along with activation of remodeling matrix (MMP2, MMP9, and TIMP1) and increasing COL1A1 mRNA expressions and collagen content increments, and most importantly, periportal fibrosis evident on histology was at maximum expressions at 24 weeks.

The strength of the present study is in the robustness as well as the novelty of the datasets, with the in vivo designs pursued to address the primary question of HSC activations and fibrogenesis in chronic drug toxicity. Additionally, the ability to demonstrate with precision the relevant pathophysiological changes in a sequential manner beginning with cell injury and finally the pathways that mediate the changes described are all too convincing. We believe this to be the first detailed morphological, functional description of development liver fibrosis, the critical component in $\mathrm{CH}$, in the setting of DILI, and the connotations of the findings are fairly wide.

In conclusion, we have been able to demonstrate that prolonged therapy with INH-RMP can lead to HSC activation and liver fibrosis in a mechanism that is dependent on oxidative stress. Our study provides initial experimental evidence to a simmering body of clinical data suggesting drugs to be important agents in $\mathrm{CH}$.

\section{Abbreviations}

$\begin{array}{ll}\text { CH: } & \text { Chronic hepatitis } \\ \text { DILI: } & \text { Drug-induced liver injury } \\ \text { INH: } & \text { Isoniazid } \\ \text { RMP: } & \text { Rifampicin } \\ \text { HSCs: } & \text { Hepatic stellate cells } \\ \text { CYP2E1: } & \text { Cytochrome P450 2E1 } \\ \text { NOX: } & \text { NADPH oxidase } \\ \text { ALT: } & \text { Alanine aminotransferase } \\ \text { PBS: } & \text { Phosphate-buffered saline } \\ \text { TG: } & \text { Triglyceride } \\ \text { GSH: } & \text { Reduced glutathione } \\ \text { GSSG: } & \text { Oxidized glutathione } \\ \text { TBARs: } & \text { Thiobarbituric acid reactive substances } \\ \text { GPx: } & \text { Glutathione peroxidase } \\ \text { SOD: } & \text { Superoxide dismutase } \\ \text { H\&E: } & \text { Hematoxylin and eosin } \\ \text { TUNEL: } & \text { Terminal deoxynucleotidyl transferase- } \\ & \text { mediated deoxyuridine triphosphate nick end } \\ & \text { labeling } \\ \alpha \text {-SMA: } & \alpha \text {-Smooth muscle actin }\end{array}$

TGF- $\beta$ : $\quad$ Transforming growth factor $\beta$

ELISA: $\quad$ Enzyme-linked immunosorbent assay

COL1A1: Collagen 1A1

TIMP: $\quad$ Tissue inhibitor of matrix metalloproteinase

MMP: $\quad$ Matrix metalloproteinase

PDGF-R $\beta$ : Platelet-derived growth factor receptor $\beta$

ROS: $\quad$ Reactive oxygen species

SDS-PAGE: Sodium dodecyl sulfate-polyacrylamide gel electrophoresis

PVDF: $\quad$ Polyvinylidene fluoride

ECL: $\quad$ Enhanced chemiluminescence

qRT-PCR: Quantitative real-time polymerase chain reaction

SD: $\quad$ Standard deviation.

\section{Data Availability}

The data used to support the findings of this study are included within the article.

\section{Disclosure}

The present work was published as an abstract in Journal of Clinical and Experimental Hepatology. The reference for the abstract is DOI 10.1016/j.jceh.2015.07.160.

\section{Conflicts of Interest}

The authors declare no conflict of interest regarding the publication of this article.

\section{Authors' Contributions}

Ayan Biswas and Suman Santra both equally contributed to this work and are co-first authors.

\section{Acknowledgments}

This study was funded by the Indian Council of Medical Research, New Delhi (Grant no. 5/8/5/2/2006-ECD-I to AS). The authors acknowledge the Director, I.P.G.M.E.\&R., Kolkata for allowing to carry out this work in the institute. They also acknowledge the sincere help of Pratap Pandit and Sudipta Chakraborty for providing the technical support to conduct this experimental study.

\section{References}

[1] R. J. Andrade, M. I. Lucena, M. C. Fernández et al., "Druginduced liver injury: an analysis of 461 incidences submitted to the Spanish Registry over a 10-year period," Gastroenterology, vol. 129, no. 2, pp. 512-521, 2005.

[2] N. Chalasani, R. J. Fontana, H. L. Bonkovsky et al., "Causes, Clinical Features, and Outcomes From a Prospective Study of Drug- Induced Liver Injury in the United States," Gastroenterology, vol. 135, no. 6, pp. 1924-1934.e4, 2008.

[3] E. Björnsson and L. Davidsdottir, "The long-term follow-up after idiosyncratic drug-induced liver injury with jaundice," Journal of Hepatology, vol. 50, no. 3, pp. 511-517, 2009. 
[4] E. S. Björnsson, O. M. Bergmann, H. K. Björnsson, R. B. Kvaran, and S. Olafsson, "Incidence, Presentation, and Outcomes in Patients With Drug-Induced Liver Injury in the General Population of Iceland," Gastroenterology, vol. 144, no. 7, pp. 1419-1425.e3, 2013.

[5] P. Hayashi and R. Fontana, "Clinical features, diagnosis, and natural history of drug-induced liver injury," Seminars in Liver Disease, vol. 34, no. 2, pp. 134-144, 2014.

[6] R. J. Andrade, M. I. Lucena, N. Kaplowitz et al., "Outcome of acute idiosyncratic drug-induced liver injury: long-term follow-up in a hepatotoxicity registry," Hepatology, vol. 44, no. 6, pp. 1581-1588, 2006.

[7] R. J. Fontana, P. H. Hayashi, J. Gu et al., "Idiosyncratic druginduced liver injury is associated with substantial morbidity and mortality within 6 months from onset," Gastroenterology, vol. 147, no. 1, pp. 96-108.e4, 2014.

[8] P. G. Aithal and C. P. Day, "The natural history of histologically proved drug induced liver disease," Gut, vol. 44, no. 5, pp. 731-735, 1999.

[9] W. G. B. Graham and G. R. Dundas, "Isoniazid-Related liver Disease," JAMA, vol. 242, no. 4, pp. 353-354, 1979.

[10] W. C. Maddrey and J. K. Boitnott, "Drug-induced chronic liver disease,” Gastroenterology, vol. 72, no. 6, pp. 1348-1353, 1977.

[11] M. Black, J. R. Mitchell, H. J. Zimmerman, K. G. Ishak, and G. R. Epler, "Isoniazid associated hepatitis in 114 patients," Gastroenterology, vol. 69, no. 2, pp. 289-302, 1975.

[12] J. R. Mitchell, H. J. Zimmerman, K. G. Ishak et al., "Isoniazid liver injury: clinical spectrum, pathology, and probable pathogenesis," Annals of internal Medicine, vol. 84, no. 2, pp. 181192, 1976.

[13] D. J. Girling, "The Hepatic toxicity of antituberculosis regimens containing isoniazid, rifampicin and pyrazinamide," Tubercle, vol. 59, no. 1, pp. 13-32, 1978.

[14] M. A. Steele, R. F. Burk, and R. M. DesPrez, "Toxic Hepatitis with Isoniazid and Rifampin: A Meta-analysis," Chest, vol. 99, no. 2, pp. 465-471, 1991.

[15] D. E. Kopanoff, D. E. Snider Jr., and G. J. Caras, "Isoniazidrelated hepatitis: a U.S. Public Health Service Cooperative Surveillance Study," The American Review of Respiratory Disease, vol. 117, no. 6, pp. 991-1001, 1978.

[16] R. A. Garibaldi, R. E. Drusin, S. H. Ferebee, and M. B. Gregg, "Isoniazid associated hepatitis: report of an outbreak," The American Review of Respiratory Disease, vol. 106, no. 3, pp. 357-365, 1972.

[17] J. Singh, P. K. Garg, and R. K. Tandon, "Hepatotoxicity due to antituberculosis therapy: clinical profile and reintroduction of therapy," Journal of Clinical Gastroenterology, vol. 22, no. 3, pp. 211-214, 1996.

[18] A. Chowdhury, A. Santra, K. Bhattacharjee, S. Ghatak, D. R. Saha, and G. K. Dhali, "Mitochondrial oxidative stress and permeability transition in isoniazid and rifampicin induced liver injury in mice," Journal of Hepatology, vol. 45, no. 1, pp. 117-126, 2006.

[19] F. Tietze, "Enzymic method for quantitative determination of nanogram amounts of total and oxidized glutathione: applications to mammalian blood and other tissues," Analytical Biochemistry, vol. 27, no. 3, pp. 502-522, 1969.

[20] O. W. Griffith, "Determination of glutathione and glutathione disulfide using glutathione reductase and 2-vinylpyridine," Analytical Biochemistry, vol. 106, no. 1, pp. 207-212, 1980.
[21] T. F. Slater and B. C. Sawyer, "The stimulatory effects of carbon tetrachloride on peroxidative reactions in rat liver fractions in vitro. Inhibitory effects of free-radical scavengers and other agents," The Biochemical Journal, vol. 123, no. 5, pp. 823-828, 1971.

[22] M. M. Bradford, "A rapid and sensitive method for the quantitation of microgram quantities of protein utilizing the principle of protein-dye binding," Analytical Biochemistry, vol. 72, pp. 248-254, 1976.

[23] R. F. Beers Jr. and I. W. Sizer, "A spectrophotometric method for measuring the breakdown of hydrogen peroxide by catalase," The Journal of Biological Chemistry, vol. 195, no. 1, pp. 133-140, 1952.

[24] D. E. Paglia and W. N. Valentine, "Studies on the quantitative and qualitative characterization of erythrocyte glutathione peroxidase," The Journal of Laboratory and Clinical Medicine, vol. 70, no. 1, pp. 158-169, 1967.

[25] A. Nandi and I. B. Chatterjee, "Assay of superoxide dismutase activity in animal tissues," Journal of Biosciences, vol. 13, no. 3, pp. 305-315, 1988.

[26] J. Bai and A. I. Cederbaum, "Adenovirus mediated expression of CYP2E1 produces liver toxicity in mice," Toxicological Sciences, vol. 91, no. 2, pp. 365-371, 2006.

[27] B. Herrera, M. M. Murillo, A. Alvarez-Barrientos, J. Beltrán, M. Fernández, and I. Fabregat, "Source of early reactive oxygen species in the apoptosis induced by transforming growth factor-beta in fetal rat hepatocytes," Free Radical Biology \& Medicine, vol. 36, no. 1, pp. 16-26, 2004.

[28] R. Bataller, R. F. Schwabe, Y. H. Choi et al., "NADPH oxidase signal transduces angiotensin II in hepatic stellate cells and is critical in hepatic fibrosis," The Journal of Clinical Investigation, vol. 112, no. 9, pp. 1383-1394, 2003.

[29] A. A. Nanji, C. L. Mendenhall, and S. W. French, "Beef fat prevents alcoholic liver disease in the rat," Alcoholism, Clinical and Experimental Research, vol. 13, no. 1, pp. 15-19, 1989.

[30] D. E. Kleiner, E. M. Brunt, M. Van Natta et al., "Design and validation of a histological scoring system for nonalcoholic fatty liver disease," Hepatology, vol. 41, no. 6, pp. 1313-1321, 2005.

[31] P. Bedossa and T. Poynard, "An algorithm for the grading of activity in chronic hepatitis C. The METAVIR Cooperative Study Group," Hepatology, vol. 24, no. 2, pp. 289-293, 1996.

[32] Q. Cao, K. M. Mak, and C. S. Lieber, "Dilinoleoylphosphatidylcholine prevents transforming growth factor-betal-mediated collagen accumulation in cultured rat hepatic stellate cells," The Journal of Laboratory and Clinical Medicine, vol. 139, no. 4, pp. 202-210, 2002.

[33] M. Mandal, A. Mandal, S. Das, T. Chakraborti, and C. Sajal, "Clinical implications of matrix metalloproteinases," Molecular and Cellular Biochemistry, vol. 252, no. 1-2, pp. 305-329, 2003.

[34] Z. N. Oltvai, C. L. Milliman, and S. J. Korsmeyer, "Bcl-2 heterodimerizes in vivo with a conserved homolog, Bax, that accelerates programmed cell death," Cell, vol. 74, no. 4, pp. 609-619, 1993.

[35] D. E. Kleiner, N. P. Chalasani, W. M. Lee et al., "Hepatic histological findings in suspected drug-induced liver injury: systematic evaluation and clinical associations," Hepatology, vol. 59, no. 2, pp. 661-670, 2014.

[36] H. Devarbhavi, R. Dierkhising, W. K. Kremers, M. S. Sandeep, D. Karanth, and C. K. Adarsh, "Single-center experience with 
drug-induced liver injury from India: causes, outcome, prognosis, and predictors of mortality," The American Journal of Gastroenterology, vol. 105, no. 11, pp. 2396-2404, 2010.

[37] R. Kumar, Shalimar, V. Bhatia et al., "Antituberculosis therapy-induced acute liver failure: magnitude, profile, prognosis, and predictors of outcome," Hepatology, vol. 51, no. 5, pp. 1665-1674, 2010.

[38] J. A. Pilheu, M. C. de Salvo, and J. A. Barcat, "Effect of isoniazid and rifampicin regimens on the liver of tuberculosis patients," Medicina, vol. 39, no. 3, pp. 298-304, 1979.

[39] J. A. Pilheu, M. C. de Salvo, O. Koch, and J. A. Barcat, "Light and electron microscopy studies of the liver in tuberculosis patients receiving rifampin and isoniazid," Medicina, vol. 41, no. 4, pp. 439-445, 1981.

[40] M. Parola and G. Robino, "Oxidative stress-related molecules and liver fibrosis," Journal of Hepatology, vol. 35, no. 2, pp. 297-306, 2001.

[41] A. Canbay, P. Taimr, N. Torok, H. Higuchi, S. Friedman, and G. J. Gores, "Apoptotic body engulfment by a human stellate cell line is profibrogenic," Laboratory Investigation, vol. 83, no. 5, pp. 655-663, 2003.

[42] S. S. Zhan, J. X. Jiang, J. Wu et al., "Phagocytosis of apoptotic bodies by hepatic stellate cells induces NADPH oxidase and is associated with liver fibrosis in vivo," Hepatology, vol. 43, no. 3, pp. 435-443, 2006. 